\title{
Autonomous vehicle navigation with semantical modelling: A project overview
}

\author{
Alessandro Correa Victorino, Marie-Hélène Abel
}

\begin{abstract}
In this paper we consider the problem related to the autonomous navigation of a robotic vehicle by modeling and describing the semantics of the context associated to the navigation. Considering the semantical modelling of the navigation context, as obstacles, pedestrians, roundabouts, information on the vehicle itself (load, power, etc.), on the robotic vehicle, it is integrating to add this symbolic artificial intelligence to a classical navigation control scheme. This semantic modeling generates an additional degree of abstraction by "reasoning" about the information collected by the various sensors of the autonomous vehicle in order to build a navigation context. This navigation context is able to provide decision support for the autonomous or semi-autonomous vehicle (interaction to the driver). In this paper, we present a project developed at Université de Technologie de Compiègne, Laboratory Heudiasyc (Heuristics, Diagnosis and Complex Systems), in France, showing initial modeling results by defining and selecting the relevant information in the creation of a context for the vehicle, structuring the contextual information so as to be able to reasoning on it and thus dynamically update the context in which the vehicle operates; maintaining information by choosing suitable data persistence models in order to be able to query previous simulations efficiently.
\end{abstract}

\section{INTRODUCTION}

The navigation of a robotic vehicle in an autonomous way, in a highway or in a local urban environment, following the traffic rules and avoiding the obstacles, requires some functionalities as perceiving the nearby environment, interacting with any spatial and temporal changes in this environment, and rescheduling its mission according to this dynamic.

Most of the current works in autonomous navigation for robotic intelligent vehicles considers the displacement of the vehicle in a local context (road monitoring, obstacle avoidance, etc.), with an onboarded navigation control loop based on the perception of the environment. In [1] visual-based navigation method is combined to an obstacle avoidance method based on the methodology of dynamic window approach. Other works consider the global navigation problem of running a complete itinerary with the management of intersections and roundabouts [2], [3]. These navigation methodologies enable the autonomous vehicle to realize a trajectory in a safely way, following the road and avoiding the obstacles, based on geometrical/physical mathematical models and automatic control methodologies, without however "understanding" the nature of these navigation contexts. Building a semantic modeling would thus make it possible to generate an additional degree of abstraction by "reasoning" about the information

*Resrach supported in part by the Idex Sorbonne Université - Labex MS2T. Authors are with the Université de technologie de Compiègne, CNRS, collected by the various sensors of an autonomous vehicle in order to build a navigation context for the latter. Ultimately, this navigation context should be able to provide decision support for the autonomous or semi-autonomous vehicle (to inform the driver).

Context is a complex definition [4]. It is the principal element applied to help intelligent agents understand how events in the surrounding world influence their behavior [5]. Its definition is limited, those presented in the literature are too dependent on their own contexts [6]. The latter depend on the field of application [7]. Some definitions are presented as, the context is the set of circumstances surrounding an event or an object [6]. This definition is widely used in the field of psychology. Context is a set of explicit variables that models contextual factors in the underlying domain, for example, time, place, environment, devices, occasion, etc. [8]. This definition comes from recommendation systems. Again, the context is the set of environmental states and parameters that determine the behavior of an application or in which an event occurs and is of interest to the user [9]. This definition is generally used in the field of contextual computing.

Our research concerns to combine the classical autonomous navigation methods applied to robotic vehicle, by considering a semantic model of the navigation context. In this way, obstacles, pedestrians, roundabouts, crossings, information on the vehicle itself (load, power, etc.) will be considered for the final control inputs computed by the navigation control loop. On the one hand, it is a question of formally defining the elements of the navigation context that we can obtain, for example from the visual perception of the road and the elements around the vehicle provided by an on-board camera or through inter-vehicles communication, and on the other hand show their use in a decision support process, for example in the context of an overtaking task, emergency stop and entering and exiting roundabouts. The on-board system will thus navigate by considering the semantic representations of the context (itself + environment) in which it operates.

In this paper we present the developments performed in this field in the Heudiasyc Lab, at the Université de Technologie de Compiègne, France, through an overview of developments and results obtained in an ongoing project. The rest of the paper is structured as follows. In section II it is presented the theoretical background associated to the proposed semantical navigation control methodology. The developments and proposals are presented in section III. We present in section IV the validation results using the professional driving simulator SCANeR

Heudiasyc (Heuristics and Diagnosis of Complex Systems), CS 60319 - 60 203 Compiègne Cedex alessandro.victorino@hds.utc.fr,marie-helene.abel@hds.utc.fr 
Studio, before presenting some conclusions and perspectives in section V.

\section{THEORETICAL BACK-GROUND}

\section{A. The context modelling}

In order to develop a semantic context model, it is necessary to identify the elements that constitute the context associated with displacement of a vehicle. We are interested in the spatial context. The latter can be divided into three dimensions:

- The static spatial context: information that can have an impact on the user's environment;

- The dynamic spatial context: georeferenced information collected by different types of sensors;

- The internal spatial context: information accessible from local devices such as GPS.

An important element to be considered in vehicle navigation is the temporality into the system. This will enable to define periods of crowds, for example. Coupling these temporal data to the static environment data would allow the vehicle to adapt its driving in function of the critical instantaneous situations as the density of traffic. This point is not considered in the current stage of our project, but it could be considered coupled to the global itinerary provided by a connected driving assistant or provided from the inter-vehicle communication network.

The context can be categorized in terms of navigation scenarios as follows, as shown in Fig. 1:

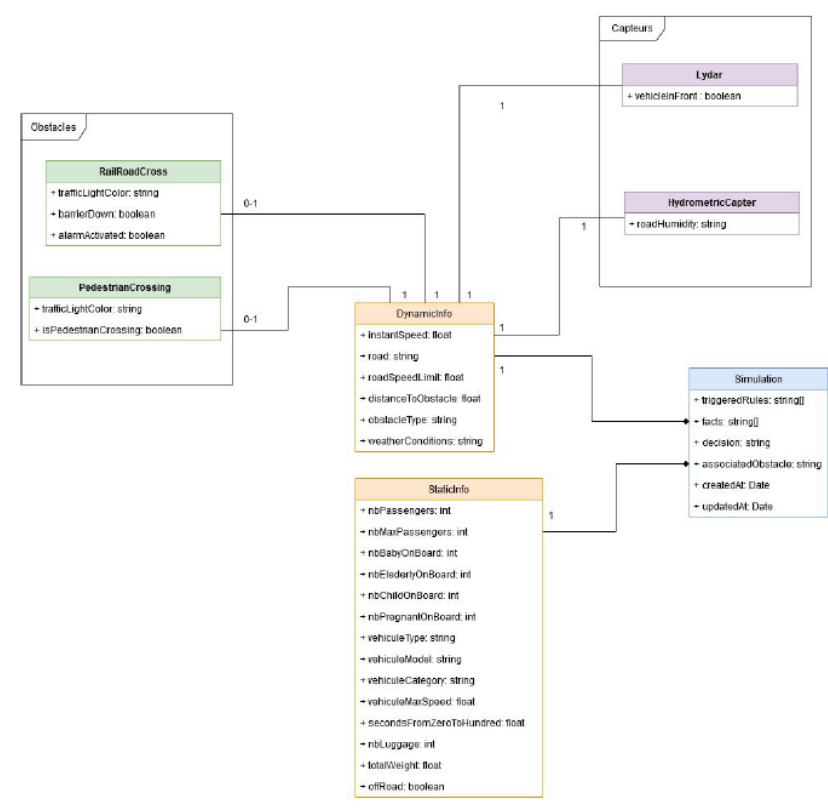

Figure 1. Exemple of the persistency of the context.

- Static information (StaticInfo class on the UML in Fig. 1): The knowleged related to the perennial elements associated to the navigation. This information can be entered in an anticipated way of the journey and may concerns:
- Type and condition of the passengers: how many people in the vehicle, health state and fragility of passengers, children in infants or pregnant women, etc.;

- Vehicle information: technical performance, model, type, etc.;

- Real time environment information acquired from onboarded sensors:

- velocity of the vehicle;

- climate information;

- traffic signs, state of the road;

- Obstacles detected in a given moment, intersections, pedestrian crossing, railroad crossing.

A current way to take this semantical information to construct the context of the vehicle navigation is using Ontologies. An ontology is a semantical technology representing the context around a situation. It is a way to represent the knowledge in a structure like that shown in Fig.1, but providing the possibility to create new knowledge from that already stored, as a result of an integrated reasoner. The elements of the context managed by an ontology has a sense for humans and for machines. A well-used language used for modelling ontologies is the OWL, the Web Ontology language, developed by Stanford University, Protégé [10]. Each information in an OWL ontology is stored as a triple, in the form Subject - Predicate - Object:

$$
\begin{gathered}
\text { MyPassenger isOfType PregnancyWomen } \\
\text { MyCar hasMaxSpeed } 70
\end{gathered}
$$

The ontology knowledge base is obtained regrouping all the storage triples in a graph (the knowledge graph). OWL Ontologies make the Open World Assumption [11], for which the statements that are not explicitly said to be true are not assumed to be false, and this allows for a better reasoning over them. Ontologies are structured in two a terminological component, called TBox, and an assertional component called ABox. The TBox contains the objects constituting the context, like EgoVehicle, Obstacle, Passenger. The TBox are also characterized by the relationships between objects or between objects and some data, the Object Properties and the Data Properties respectively.

\section{B. The robotic navigation control loop}

On the other side, autonomous vehicle navigation control loop needs to cope to some functionalities, like: environment perception, mapping and localization, motion planning, decision and control. Through images captured by one or more cameras, LIDAR and other useful sensors, the perception task is designed to detect and understand the local environment where the vehicle is driving. Some researches comprising the perception subject are found in [12], [13] and [14]. An optimal collision free path, from the current vehicle state to the desired state are generated by motion planning methods, and can be divided into global planners or local planners, where local information is considered. These methods consist on candidate trajectories calculation based on kinodynamic model, and then selecting the best one considering the safeness and others relevant assumptions, such methods were presented in some 
DARPA Urban Challenge team cars [15]. A classic reactive motion planning DWA proposed by [16], evaluates pair of velocities selection by an objective function optimization, considering a short time and descanting any velocities which generates path with collision according to a minimum distance.

Next section reviews some developments obtained at Heudiasyc Lab in order to provide to an autonomous robotic vehicle the possibility of reasoning about the semantical context of navigation around it.

\section{THE HEUDIASYC PROPOSAL: A PROJECT REVIEW}

We have put the problem in terms of developing a context model, based on the ontology structure and then to integrate the reasoning about the context to the navigation control loop of the robotic vehicle. The project presentation is structured in terms of control navigation methodology and the context modelling.

\section{A. Autonomous navigation control}

The control methodology used to autonomous navigate the vehicle is the lane keeping and obstacle avoidance method developed in our previous work [1]. The motions of the robotic vehicle, equipped with onboarded camera and LiDAR perceiving the road, are controlled in order to keep the lane by applying a visual-servoing technique [17] and avoiding the eventual obstacles by applying the Image-Based Dynamic Window Approach (IDWA) [18]. The method is resumed recalled here, but details can be found in our previous work [1]:

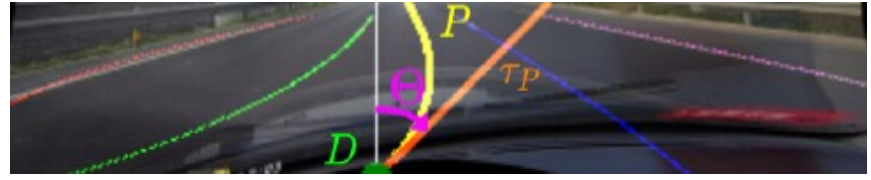

Figure 2. Image Features; point $(\mathrm{D})$, path $(\mathrm{P})$, path tangent $(\tau P)$, angle between $\tau P$ and $y$-axis $(\theta)$, others lines represents lane boundaries detected.

1) Visual Servoing: The visual servoing allows defining the vehicle commands from feature images. From the lane detection, we can evaluate if the car is in the center of the lane or not, this evaluation can be defined by an error illustrating if we are very far from the center or not. Thus, we can calculate the command to keep the vehicle in its lane. In our case features image, 's' is defined by the point ' $\mathrm{D}=(\mathrm{X} ; \mathrm{Y})$ ', which represents the intersection of the path to follow the border of the image, and the angle ' $\theta$ ', which represents the angle between the tangent ' $\tau P$ ' of the path ' $\mathrm{P}$ ' evaluated in ' $\mathrm{D}$ ' with the ordinate axis. Fig. 2 represents these features on an example. All vision-based control aims to minimize an error ' $\mathrm{e}(\mathrm{t})$ ' defined as follow:

$e=s(t)-s^{*}$

where ' $s$ ' features at the ' $t$ ' instant and ' $s$ "' desired values of these features. From this error, we can define row controller and column controller allowing us to reduce the error ' $\mathrm{e}$ '.

2) Image Based Dynamic Window Approach: The dynamic window approach (DWA) is an online collision avoidance strategy, which means this strategy is based on the current states of the robot (i.e. current dynamics of the robot), that defines the best velocity. The IDWA method is divided into two steps:

- Defining the limitation of the search space, the velocities that can be reached by the robot, and that guarantee not to collide with obstacles;

- Finding the velocity maximizing the objective function.

The initial objective function is defined as follow:

$\mathrm{G}(\mathrm{v}, \mathrm{w})=\alpha^{*}$ heading $(\mathrm{v}, \mathrm{w})+\beta^{*} \operatorname{dist}(\mathrm{v}, \mathrm{w})+\gamma^{*} \operatorname{velocity}(\mathrm{v}, \mathrm{w})$

Where, function 'heading' evaluates if the execution of the command $(\mathrm{v} ; \mathrm{w})$, computed from the visual servoing control technique, allows to stay in the center of the lane. The function 'dist' evaluates if we stay at a certain distance from the obstacles around the robot and finally the function 'velocity' evaluates if the robot moves at the desired speed.
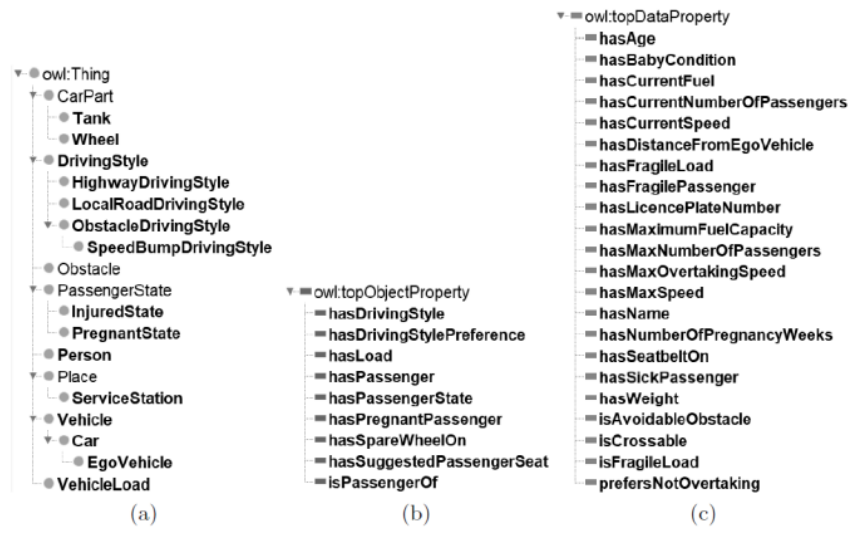

Figure 3. Modelling the context of navigation with. (a) Classes; (b) Object Properties; (c) Data Properties.

\section{B. Vehicle navigation context modelling}

The modelling of the semantical context around the autonomous vehicle navigation proposed in the Heudiasyc project, in works performed by our master student F. Faruffini [19], is structured with an ontology in the OWL language, integrating different levels of contextual information: Passenger-related information, road-related information, loadrelated information, robot-related information, obstacle information and other information. All this information is structured in terms of classes, data properties and object properties as depicted by Fig. 3.

1) Passenger-related information are related to the different driving styles, in a faster and sport mode or in a smooth mode. Related to the different level of comfort, associated to the maximal accelerations or jerk defined. Related to the preferences in the itinerary, as local road, highways, etc. Each passenger can set its preferences that will be registered in an object.

2) Road-related information will make possible to model the road speed limit, the sense of circulation, specific traffic rules, and other knowledge associated to the road. This information 
can be simply recovered from onboarded sensors perceiving road signs, or through some digital map as OpenStreetMap.

3) Load-related information provides to the robotic vehicle the input useful to adapt its driving conditions with respect to the load specifications. If the robot is carried out a fragile load it will overtake an obstacle differently if it is transporting a non-fragile load. The weight of the transported objects will make possible to adjust the acceleration and distance to stop.

4) Robot-related information concerns the physical model of the vehicle, as the available power level (fuel or battery), the number and type of tires, available passenger places and current occupied places. This information is useful for the security of the navigation.

5) Obstacle information model the characteristics of the possible obstacles detected and recognized by the onboarded perception system of the robotic vehicle, as its mass and material, making possible a label assignation, as static or moving objects.

6) Other information will model objects that are not considered in any of the previous instances, as meteorological conditions, temporally events on the road (manifestations, works, etc).

Fig. 2 presents a version of the developed navigation ontology, considering the instances listed above. It is obviously not complete, but useful for proving the pertinence of our contextual autonomous navigation proposal.

\section{B. Reasoning basis}

The reasoning basis is a set of rules running on the navigation ontology, that will lead to a specific behavior of the robotic vehicle that corresponds to a predefined constraint modelled in the ontology (through the contextual information listed at the above paragraph). In our project we decided to use the SWRL - the Semantic Web Rule Language - and the chosen reasoner as in [21].

Let's consider an instance Jean, who is a passenger preferring a slower speed than the maximum one fixed by the traffic rules, and MyCar as our ego vehicle can define the following triples:

$$
\begin{gathered}
\text { MyCar hasPassenger Jean } \\
\text { MyCar hasDrivingStyle StandardDrivingStyle } \\
\text { StandardDrivingStyle hasMaxSpeed } 50
\end{gathered}
$$

and

\section{Jean hasDrivingStylePreference JeanDrivingPreference JeanDrivingPreference hasMaxSpeed 30}

A SWRL rule to adapt the maximum speed of the car to the one of the passengers can be defined as:

$$
\begin{gathered}
\text { EgoVehicle }(? v)^{\wedge} \text { hasPassenger }(? v ; ? p)^{\wedge} \text { Person }(? p)^{\wedge} \\
\text { hasDrivingPreference }(? p ; ? \text { pref }) \rightarrow \\
\text { hasDrivingStyle }(? v ; ? \text { pref })
\end{gathered}
$$

This rule will provide to the navigation control loop of the robotic vehicle an information concerning the speed limitation considering the preferences of the passenger Jean. Next section presents a proposal of how to merge the reasoner driving recommendations to the autonomous navigation control loop.

\section{Autonomous navigation control with context information}

We have proposed two schemas in order to integrate the semantical context information, from the ontology and reasoner basis, to the navigation control loop of the robotic vehicle: (i) integrating a Decision block to the navigation control loop, as showed in Fig. 4; (ii) Directly integrating the reasoner recommendations in the IDWA control strategy of the navigation loop, as shown in Fig. 5.

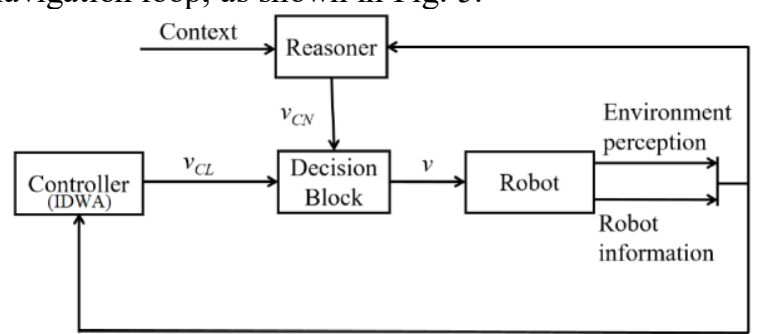

Figure 4. $\quad V c l$ is the control input that is normaly applied to the robot without context awareness; $V c n$ is the recommendation from the reasoner; $V$ is the final control imput to the robot.

In this navigation control schemes, for simplicity, we consider as control input only the longitudinal velocity to be applied to the vehicle.

1) Integrating a decision block, shown in Fig. 4, considers the recommended velocity by the context reasoner modulating the automatic navigation control input, in the "Decision block". The final control input proposed in [19] is defined with a convex combination of the control input from the control loop $(V c l)$ and the control imput from the context navigation $(\mathrm{Vcn})$,

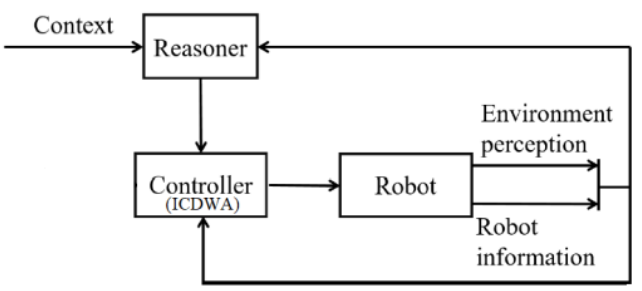

Figure 5. Directely integrating the recommandation from the context reasoner to the navigation control loop.

$$
V=\left\{\begin{array}{cl}
V c l, & V c l<V c n \\
\gamma * V c l+(1-\gamma) * V c n & V c l>V c n
\end{array}\right.
$$

where $\gamma \in[0,1]$. The value of $\gamma$ is a function of the the traffic conditions and needs to be defined in real-time. This scheme is more simple to be programmed under real-time constraints.

2) Directly integrating the reasoner recommendations in the IDWA, as indicated in Fig.5, is an improvement of the previous blending control rule, which considers a new proposal of the IDWA in order to take into account the context recommendation, as we proposed in [20]. The novelty consists in considering a new cost function in the original DWA 
methodology, that contributes in the total optimization of the objective fonction in eq. (1). Let us consider this new DWA objetive fonction as,

$$
\begin{array}{r}
\mathrm{G}(\mathrm{v}, \mathrm{w})=\alpha^{*} \text { heading }(\mathrm{v}, \mathrm{w})+\beta^{*} \operatorname{dist}(\mathrm{v}, \mathrm{w})+\gamma^{*} \operatorname{velocity}(\mathrm{v}, \mathrm{w})+ \\
\delta * \operatorname{context}(\mathrm{v}, \mathrm{w})
\end{array}
$$

With $\delta \in \mathbb{R}$. We called this new method, ICDWA (Imageand-Context-based Dynamic Window Approach) [20]. This new approach considers the longitudinal velocity " $\mathrm{v}$ " and the yaw velocity " $w$ " as control navigation inputs to the robotic vehicle, so, in this case, the context reasoner recommends (Vcn,Wcn) as context navigation inputs. The optimization objective is to find $(\mathrm{v}, \mathrm{w})$ that maximizes $\mathrm{G}(\mathrm{v}, \mathrm{w})$. Then, and considering the context term in $G$, the expression of context $(\mathrm{v}, \mathrm{w})$ must be defined so that this forth term of $\mathrm{G}$ is maximized when $(\mathrm{v}, \mathrm{w})$ goes to the recommended $(\mathrm{Vcn}, \mathrm{Wcn})$. We proposed to define this context cost function as,

$$
\operatorname{context}(\mathrm{v}, \mathrm{w})=\varepsilon^{*}(\operatorname{context}(\mathrm{v})+\operatorname{context}(\mathrm{w}))
$$

where the two functions context(v) and context(w) considers the cost associated to the logitudinal and yaw velocity separetely. The weight $\varepsilon \in[0,1]$ indicates the importance of the context recommendation in the total optimization procedure. Let's consider in this explanation only the longitudinal velocity criteria, the formulation to the yaw velocity is similar, and considering context(v) defined in terms of the sigmoid function,

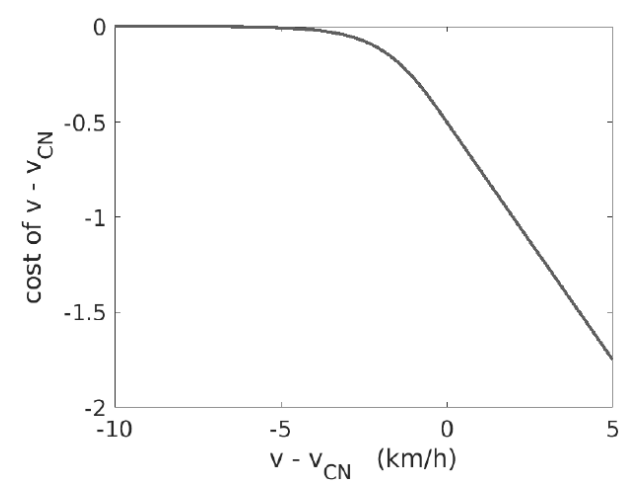

Figure 6. The evolution of the cost context(v), when varrying the longitudinal velocity $v$ in the optimization process.

$$
\sigma(\mathrm{x})=\frac{1}{1+e^{-x}}
$$

$$
\dot{\sigma}(\mathrm{x})=\sigma(\mathrm{x})^{*}(1-\sigma(\mathrm{x}))
$$

putting $\mathrm{x}=\mathrm{v}-\mathrm{Vcn}$, the context(v) cost is defined as,

$\operatorname{context}(\mathrm{v})=\left\{\begin{array}{cc}-\sigma(x) & x \leq 0 \\ -(\sigma(0)+\dot{\sigma}(0) * x) & x>0\end{array}\right.$

by computing $\sigma(0)$ and $\dot{\sigma}(0)$ from eq. 4 , we have

$\operatorname{context}(\mathrm{v})=\left\{\begin{array}{cc}-\sigma(x) & x \leq 0 \\ -\frac{1}{2}-\frac{1}{4} * x & x>0\end{array}\right.$

Then we will have high values of context $(v)$ when $v<V c n$, and lower values when $v<V c n$, as shown in Fig. 6.

\section{RESULTS AND VALIDATIONS}

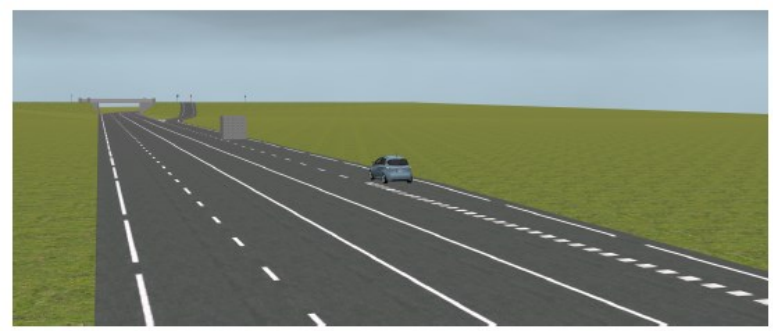

Figure 7. Autonomous driving in the SCANeR Studio.

We review here the results presented in [19] and in [20] in order to show the application of the context modelling and reasoning in the autonomous navigation of a robotic vehicle. We have onboarded our IDWA navigation control method, as described in section III-A in the professional driving simulator SCANeR Studio, Fig. 7, acquired many autonomous navigation data, and then proceeded to the evaluation of the ICDWA method proposed in III-C. First of all, we present here results using the first methodology, recommend input modulating the control loop input, presented in section III.C.1 for an overtaking situation. Fig. 8 shows the final input velocity applied to the robotic vehicle. The solid line shows the evolution without context awareness, the other lines represent the evolution of the velocity when considering the speed preferences of the passenger, with different values of $\gamma$.

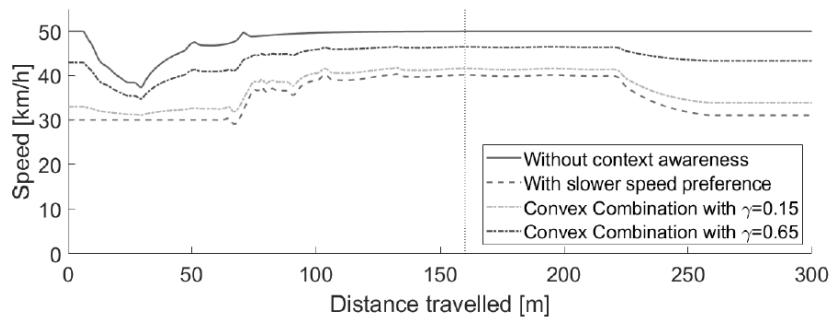

Figure 8. Context modulation in the velocity control.

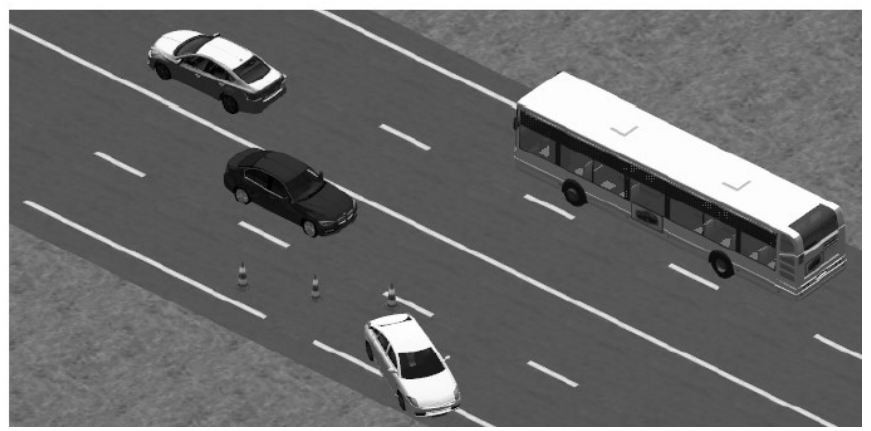

Figure 9. Autonomous avoiding static obstacles in the lane.

We can see the influence of the passenger preference on the final control input applied to the robotic vehicle.

The second validation test was performed using the second methodology recalled in section III.C.2. In this case, the autonomous navigation loop onboarded in SCANeR Studio was the new methodology ICWDA proposed in [20]. The navigation scenario consists of an autonomous avoidance of static obstacles in the lane, with a lane change, as shown in 
Fig. 9. It is considered that the passenger speed preference is $10 \mathrm{~km} / \mathrm{h}$ in this example. Parameters of objective functions in (2) and (3) were respectively set as $\alpha=1, \beta=0.3$ and $\gamma=$ 0.1 . Fig. 10 shows 3 tests realized in this scenario: with no semantical context modelling intervention (continuous line), the last two with different values of $V c n$ recommendations and weight parameter.

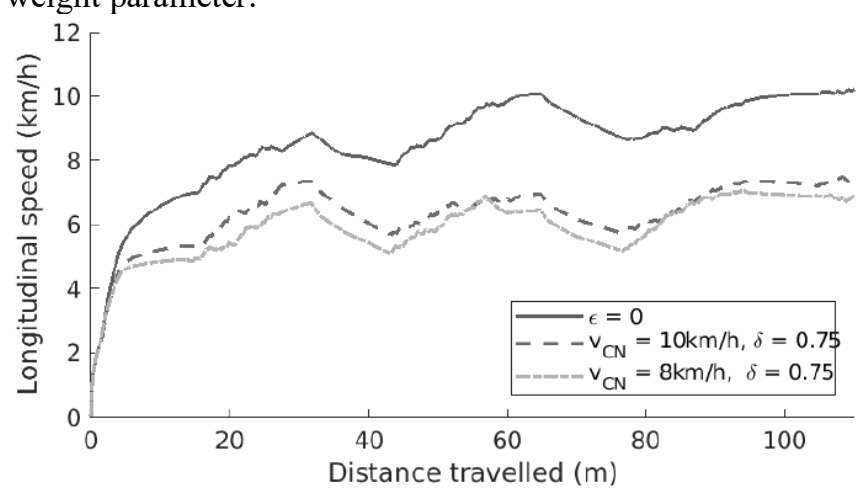

Figure 10. Evaluation of the ICDWA methodology.

Figure 11. The first test is the one in which we

It is shown the decelerations when the obstacles are detected and accelerations during the overtaking maneuvers. We can see again that the final control input computed with the ICDWA methodology, promote the lane keeping with obstacle avoidance, and, in addition, respect the passenger preferences. Besides, we can conclude that the values of tuning parameters as $\alpha, \beta, \gamma$ and $\varepsilon$ need to be carefully chosen, because they could affect considerably the performance of the system. As shown in figure, the tuned value of the $\gamma$ prevents the system to reach the maximal desired velocity preferred by the passenger.

\section{CONCLUSIONS AND PERSPECTIVES}

This paper discussed the evolution of the project going in the Heudiasyc laboratory, Université de Technologie de Compiègne in France, concerning the consideration of the semantical modelling of the context around the navigation to control the autonomous displacement of a robotic vehicle. The paper was structured as a project survey, by recalling the principal points of the proposed methodology and results. We presented a methodology to model the context using Ontologies and a reasoner basis model [19]. This context structure was, then, used in association to an autonomous vehicle navigation methodology, based on the IDWA method [1], generating a new robotic vehicle navigation methodology called ICDWA [20]. Validation results proved the pertinence of our method, leading us going forward if our researches in how to making the autonomous vehicle "sensible" to the context involving the navigation task.

Next steps concern the completion of the ontology by modelling new classes, objects and data related to the vehicle navigation. Evaluating the rule generation in the context reasoner in order to cope to the real-time dynamic changes in the context. New challenges will appear when testing the method in our real experimental robotic vehicle.

\section{REFERENCES}

[1] Danilo Alves Lima, Alessandro C Victorino. "A hybrid controller for vision-based navigation of autonomous vehicles in urban environments". IEEE Transactions on Intelligent Transportation Systems, vol. 17, no. 8, pp. 2310-2323, August 2016.

[2] E. Pereira, D. Alves de Lima, A. Victorino. Autonomous vehicle global navigation approach associating sensor based control and digital maps. IEEE International Conference on Robotics and Biomimetics (ROBIO’2014), Bali, Indonesia, December 2014.

[3] Ide-Flore Kenmogne, Danilo Alves de Lima and Alessandro Correa Victorino. "A Global Navigation Management Architecture applied to Autonomous Robots in Urban Environments". In : 18th International IEEE Conference on Intelligent Transportation Systems (ITSC'15), Canary Islands, Spain, Septembre 2015

[4] Adomavicius G. \& Jannach D. Preface to the special issue on contextaware recommender systems. User Modeling and User-Adapted Interaction, volume 24, p. 1-5: Springer.

[5] Kofod-Petersen A. \& Cassens J. Using activity theory to model context awareness. In Modeling and Retrieval of Context, p. 1-17: Springer, Berlin, Heidelberg, 2006.

[6] Bazire M. \& Brézillon P. Understanding context before using it. In International and Interdisciplinary Conference on Modeling and Using Context, p. 29-40: Springer, Berlin, Heidelberg, 2005.

[7] Palmisano C., Tuzhilin A. \& Gorgoglione M. Using context to improve predictive modeling of customers in personalization applications. IEEE transactions on knowledge and data engineering, volume 20 , number 11 , p. $1535-1549$.

[8] Adomavicius G. \& Tuzhilin A. Context-Aware Recommender Systems. Recommender Systems Handbook, p. 217, 2011.

[9] Chen G. \& Kotz D A survey of context-aware mobile computing research. Technical Report. Dartmouth College, Hanover, NH, USA. 2000 .

[10] M. A. Musen, "The protégé project: a look back and a look forward," AI Matters, vol. 1, no. 4, pp. 4-12, 2015.

[11] S. Russell and P. Norvig, Artificial Intelligence: A Modern Approach. USA: Prentice Hall Press, 3rd ed., 2009.

[12] Li, Y., \& Ibanez-Guzman, J. (2020). Lidar for Autonomous Driving: The principles, challenges, and trends for automotive lidar and perception systems. Retrieved from http://arxiv.org/abs/2004.08467

[13] Zhao, G., Xiao, X., Yuan, J., \& Ng, G. W. (2014). Fusion of 3D-LIDAR and camera data for scene parsing. Journal of Visual Communication and Image Representation, 25(1), 165-183. https://doi.org/10.1016/j.jvcir.2013.06.008

[14] Van Brummelen, J., O’Brien, M., Gruyer, D., \& Najjaran, H. (2018).

[15] Urmson, C.; Anhalt, J.; Bagnell, D.; Baker, C.; Bittner, R.; Clark, M.N.; Dolan, J.; Duggins, D.; Galatali, T.; Geyer, C.; et al. Autonomous driving in urban environments: Boss and the Urban Challenge. J. Field Robot. 2008, 25, 425-466

[16] Arras, K.,Persson,J.,Tomatis,N., Siegwart,R.(2002).Real time obstacle avoidance for polygonal robots with a reduced dynamic window. IEEE International Conference on Robotics and Automation, volume 3, pages $3050-3055$.

[17] A. Cherubini, F. Chaumette, and G. Oriolo, "An image-based visualservoing scheme for following paths with nonholonomic mobile robots,"in2008 10th International Conference on Control, Automation, Robotics and Vision. IEEE, 2008, pp. 108-113.

[18] D. Fox, W. Burgard, and S. Thrun, "The dynamic window approachto collision avoidance,'IEEE Robotics Automation Magazine, vol. 4,no. 1, pp. 23-33, 1997.

[19] Faruffini, F., Correa Victorino, A., Abel, M.-H. "Towards a Semantic Model of the Context of Navigation". International Conference in Information and Knowledge Systems (ICKS'2021). June 2021.

[20] Faruffini, F., Correa Victorino, A., Abel, M.-H. " Context Modelling applied to the Intelligent Vehicle Navigation". IEEE 47th Annual Conference of the IEEE Industrial Electronics Society (IES). Oct 2021.

[21] Sirin, E., Parsia, B., Grau, B.C., Kalyanpur, A., Katz, Y.: Pellet: A practical owl-dl reasoner. Journal of Web Semantics 5(2),51-53(2007). 